\title{
Archéopages
}

Archéopages Archéologie et société

$45 \mid 2017$

De la terre au pot

\section{«Pains » de brai de bouleau sur un site gaulois}

Sandra Sicard

\section{OpenEdition}

Journals

Édition électronique

URL : https://journals.openedition.org/archeopages/3563

DOI : 10.4000/archeopages.3563

ISSN : 2269-9872

\section{Éditeur}

INRAP - Institut national de recherches archéologiques préventives

\section{Édition imprimée}

Date de publication : 1 mars 2018

Pagination : 138-139

ISSN : 1622-8545

\section{Référence électronique}

Sandra Sicard, « «Pains » de brai de bouleau sur un site gaulois », Archéopages [En ligne], 45 | 2017, mis en ligne le 01 janvier 2020, consulté le 11 juin 2021. URL : http://journals.openedition.org/ archeopages/3563; DOI : https://doi.org/10.4000/archeopages.3563 


\section{«Pains » de brai de bouleau}

Sandra Sicard

Inrap, UMR 6566 «Creaah», responsable d'opération

Site

Les Vairies

Saint-Sauveur-des-Landes

Ille-et-Vilaine

Date

Automne 2012

Surface

$$
2,5 \text { ha }
$$

Équipe

Analyse fluorescence $X$

Guirec Querre

ministère de la Culture et de la

Communication, UMR 6566

"Creaah"

Analyse physico-chimique

laboratoire

Nicolas Garnier
Le site gaulois des « Vairies » fouillé en 2012 est un établissement rural composé d'enclos emboités sur une surface d'au moins 1,8 ha (Sicard et al., 2016) [ill. 1]. Il est implanté au III $^{\mathrm{e}}$ siècle avant notre ère en zone humide, sur la rive gauche d'un chenal qui constitue alors la façade occidentale de l'enclos périphérique. $\mathrm{Au} \mathrm{I}{ }^{\mathrm{er}}$ siècle avant notre ère, la baisse importante du régime hydrique de ce chenal conduit au colmatage partiel du talweg qui devient une zone palustre. L'occupation se développe alors sur l'autre rive et un nouvel enclos de partition interne est installé dans la partie occidentale du site.
Dans le remplissage du fossé oriental de ce nouvel enclos, cinc petits objets groupés et disposés à la verticale ont été mis au jour [ill. 2]. Malgré leur nature organique, leur conservation a été possible grâce au fait qu'ils étaient cuits et enfouis en milieu humide. Une analyse radiocarbone effectuée sur des microrestes végétaux issus d'un de ces « pains » indique la date de 2070 $\pm 30 \mathrm{OP}(-170$ à $10 \mathrm{BC})$, ce qui confirme la contemporanéité de ces objets avec le fossé d'enclos attribué à la phase la plus récente de l'occupation.

Ces objets ont des poids (moins de $150 \mathrm{~g}$ ) et des gabarits très proches de forme allongée, de couleur brune, ils ont un aspect bombé et portent des craquelures. En coupe, ils sont compacts, de couleur marron foncé avec des inclusions beiges, ont un aspect cireux marqué par des «trainées noires » [ill. 3]. Plusieurs analyses ont été réalisées pour déterminer leur nature, indéfinissable d'après ces seules observations macroscopiques [ill. 4]. Une première détermination de composition par Fluorescence X a permis de constater que l'objet était constitué de matière organique (carbone et oxygène), la fraction minérale étant de moins de $20 \%$.
Une seconde analyse a été réalisée par microscope électronique à balayage, révélant une structure très homogène, dense et opaque, dans laquelle des restes de structures végétales sont ponctuellement visibles. Une dernière analyse physico-chimique a été entreprise. Il s'agit d'une méthodologie simplifiée, en une seule étape, où l'échantillon est traité par chromatographie en phase gazeuse couplée à la spectrométrie de masse (GC-MS). Elle a montré que l'échantillon est un extrait organique concentré, de couleur brun sombre, dont l'analyse, par la présence des biomarqueurs et des marqueurs de dégradation thermique du bouleau, a permis d'identifier du brai de bouleau.

Le brai de bouleau est une colle ou résine obtenue à partir d'écorce de bouleau chauffée à haute température : l'écorce est placée dans un contenant tel qu'une céramique trouée dans le fond et disposée sur un autre contenant qui servira à recueillir la substance liquide obtenue par la chauffe de l'écorce. Cette substance s'épaissit en refroidissant, jusqu'à devenir dure. À la chauffe, elle redevient liquide, ce qui permet de l'utiliser à nouveau. Les pains de brai de

\section{Phase 1 (Tène moyenne)}

Phase 2 (transition Tène moyenne/Tène finale)

Phase 3 (Tène finale)

Phase 3 récente (fin Tène finale)

Occupations postérieures à la période gauloise

Chemin actuel

Structure non fouillée

Localisation des pains de brai de bouleau

$\stackrel{N}{\Lambda}$

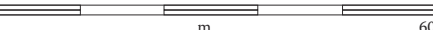

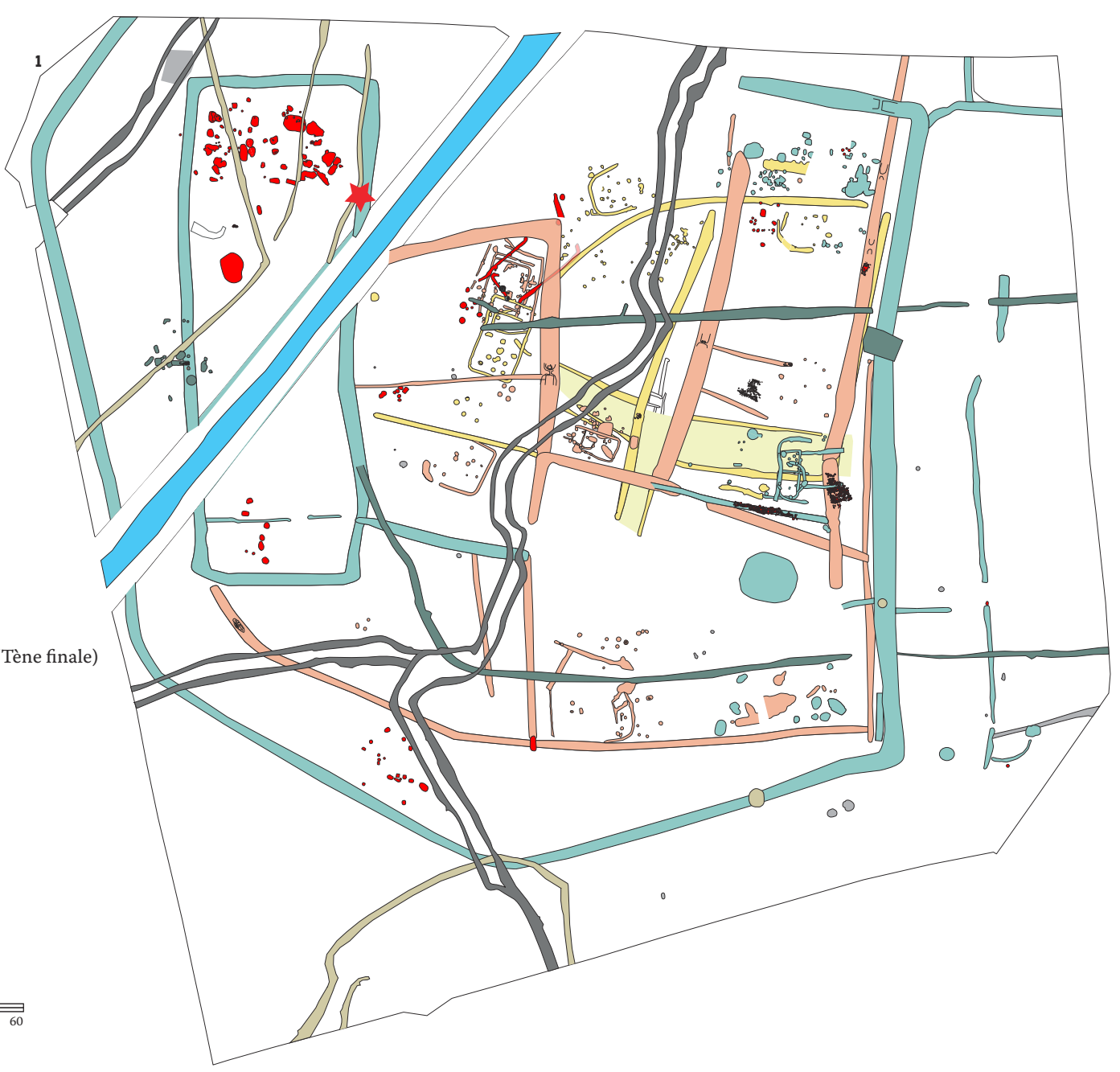



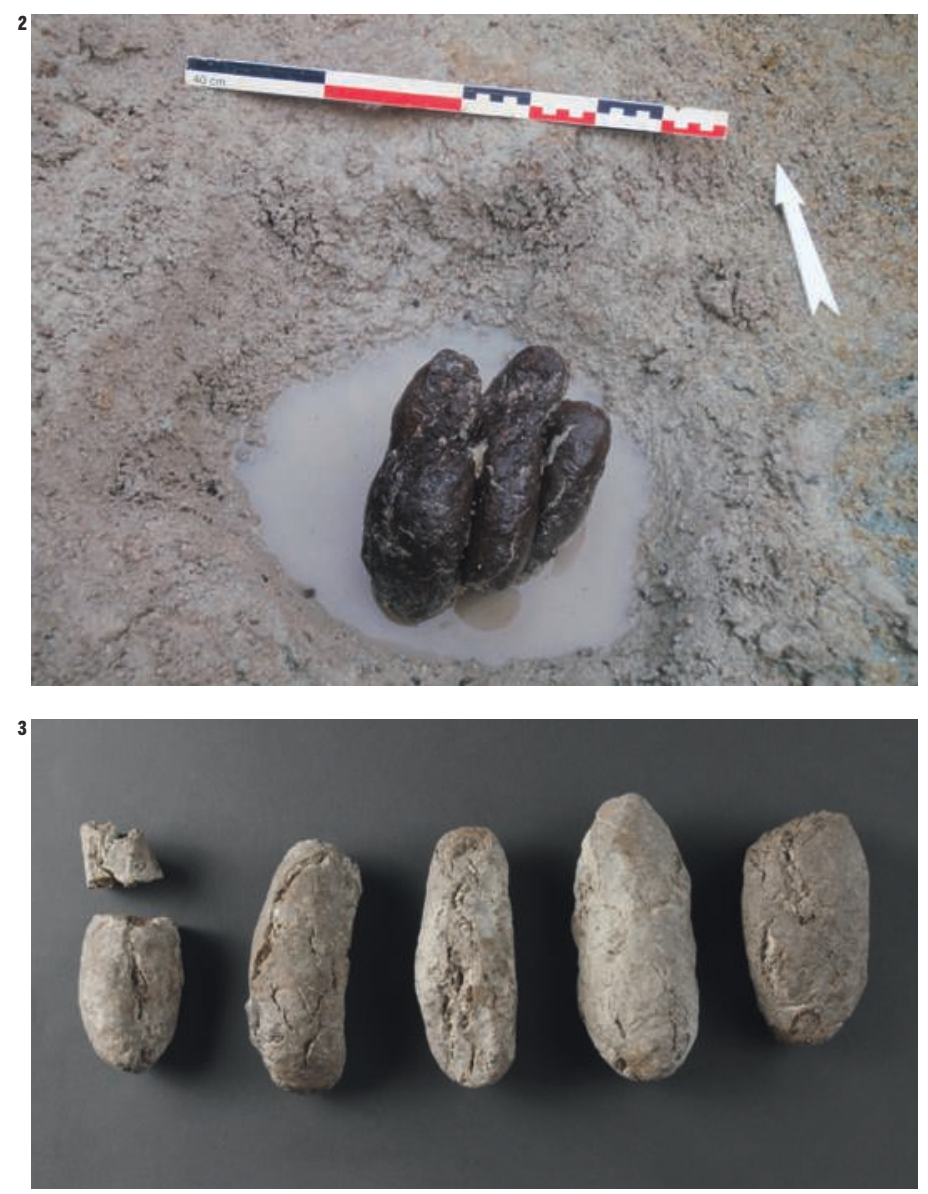

$\underset{0}{\rightleftharpoons} \stackrel{\mathrm{cm}}{\rightleftharpoons}$
2. Les pains de brai lors de leur découverte. Ils devaient initialement se trouver dans un contenant périssable, ce qui les a maintenus dans cette position originale.
3. Les pains de brai après séchage. Le plus à gauche, abîmé à une extrémité, a été coupé en deux pour analyses. 4. Un des pains de brai coupé en deux pour la réalisation d'une lame mince.

5. Vase balustre mis au jour dans le même fossé que les pains de brai.

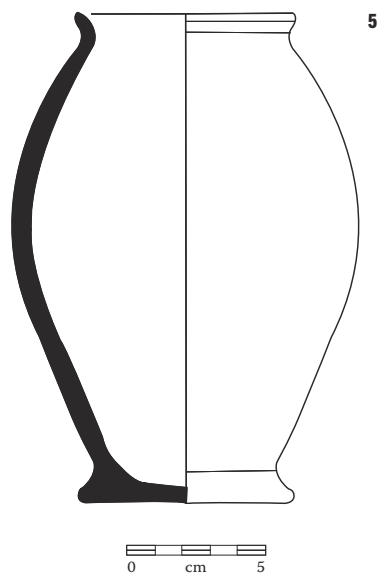

Saint-Sauveur constituaient donc des réserves utilisables en fonction des besoins en réchauffant la matière. Le fossé qui a livré les «pains » de brai de bouleau a également fourni un vase balustre complet qui a conservé sur sa paroi externe des traces de chauffe visibles, avec une altération de la partie basse du récipient probablement due à son exposition prolongée auprès d'une source de chaleur [ill. 5]. La paroi interne est, elle, intégralement tapissée d'un résidu organique épais correspondant probablement à du brai. Des analyses chimiques complémentaires des résidus conservés permettraient de confirmer leur nature.

Le brai de bouleau est connu dès l'époque préhistorique et utilisé comme colle, enduit ou adhésif (Regert, Vacher, 2001; Regert et al., 2003). Il aurait également des propriétés biocides, antiseptiques et pesticides. Aux Vairies, on peut supposer que ses propriétés d'imperméabilisant ou d'étanchéification ont dû aussi être exploitées dans un environnement particulièrement humide. L'utilisation du brai à l'époque gauloise et au début de la période romaine semble plus

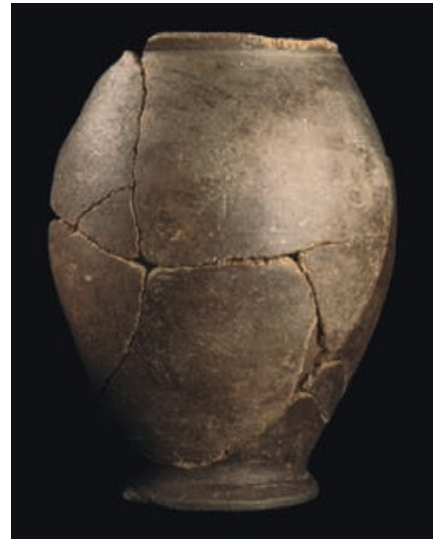

$\underset{0}{\rightleftharpoons} \rightleftharpoons$

anecdotique qu'au cours des périodes précédentes. Les exemples dans le nord-ouest de la France sont peu nombreux et les traces de brai sont identifiées comme résidus adhérant dans les céramiques ou sous forme de petits agrégats libres dans le sédiment. De tels vestiges organiques amorphes ne sont pas connus sous forme de « réserve » de matière première. En l'état actuel des connaissances, il n'existe pas d'équivalent régional aux pains de brai de bouleau des Vairies. Il s'agit donc d'une découverte exceptionnelle dont l'exploitation scientifique est encore en cours.

Références bibliographiques

REgERT M, VACHER S, 2001, «Des adhésifs organiques sur un site de La Tène au Grand Aunay (Sarthe) ", Archéopages, ${ }^{\circ} 4$, p. 20-29.

Regert M., Vacher S., Moulherat C. Decavallas O., 2003, « Adhesive production and pottery function during the Iron age at the site of Grand Aunay (Sarthe, France) ", Archaeometry, 45, $\mathrm{n}^{\circ}$ 1, p. 101-120.

SICARD S. et al., 2016, Un établissement rural gaulois en milieu humide. ZAC des Vairies, Les Croisés, Saint-Sauveur-des-Landes, Ille-et-Vilaine, rapport d'opération, Inrap-SRA Bretagne, 2 vol., 919 p. 